BABAR-PUB-03/007

SLAC-PUB-9690

hep-ex/0303036

March 2003

\title{
Evidence for $B^{+} \rightarrow J / \psi p \bar{\Lambda}$ and Search for $B^{0} \rightarrow J / \psi p \bar{p}$
}

B. Aubert,${ }^{1}$ R. Barate,${ }^{1}$ D. Boutigny,${ }^{1}$ J.-M. Gaillard,${ }^{1}$ A. Hicheur,${ }^{1}$ Y. Karyotakis,${ }^{1}$ J. P. Lees,${ }^{1}$ P. Robbe,${ }^{1}$ V. Tisserand,${ }^{1}$ A. Zghiche, ${ }^{1}$ A. Palano, ${ }^{2}$ A. Pompili, ${ }^{2}$ J. C. Chen,${ }^{3}$ N. D. Qi,${ }^{3}$ G. Rong, ${ }^{3}$ P. Wang, ${ }^{3}$ Y. S. Zhu,${ }^{3}$ G. Eigen, ${ }^{4}$ I. Ofte, ${ }^{4}$ B. Stugu, ${ }^{4}$ G. S. Abrams, ${ }^{5}$ A. W. Borgland, ${ }^{5}$ A. B. Breon, ${ }^{5}$ D. N. Brown, ${ }^{5}$ J. Button-Shafer,${ }^{5}$ R. N. Cahn, ${ }^{5}$ E. Charles,${ }^{5}$ C. T. Day,${ }^{5}$ M. S. Gill,${ }^{5}$ A. V. Gritsan,${ }^{5}$ Y. Groysman,${ }^{5}$ R. G. Jacobsen,${ }^{5}$ R. W. Kadel,${ }^{5}$ J. Kadyk,${ }^{5}$ L. T. Kerth, ${ }^{5}$ Yu. G. Kolomensky, ${ }^{5}$ J. F. Kral, ${ }^{5}$ G. Kukartsev,${ }^{5}$ C. LeClerc,${ }^{5}$ M. E. Levi, ${ }^{5}$ G. Lynch, ${ }^{5}$ L. M. Mir,${ }^{5}$ P. J. Oddone,${ }^{5}$ T. J. Orimoto, ${ }^{5}$ M. Pripstein,${ }^{5}$ N. A. Roe,${ }^{5}$ A. Romosan,${ }^{5}$ M. T. Ronan, ${ }^{5}$ V. G. Shelkov, ${ }^{5}$ A. V. Telnov, ${ }^{5}$ W. A. Wenzel, ${ }^{5}$ T. J. Harrison, ${ }^{6}$ C. M. Hawkes,${ }^{6}$ D. J. Knowles, ${ }^{6}$ R. C. Penny, ${ }^{6}$ A. T. Watson, ${ }^{6}$ N. K. Watson, ${ }^{6}$ T. Deppermann, ${ }^{7}$ K. Goetzen, ${ }^{7}$ H. Koch,${ }^{7}$ B. Lewandowski, ${ }^{7}$ M. Pelizaeus,${ }^{7}$ K. Peters, ${ }^{7}$ H. Schmuecker ${ }^{7}$ M. Steinke, ${ }^{7}$ N. R. Barlow,${ }^{8}$ W. Bhimji, ${ }^{8}$ J. T. Boyd,${ }^{8}$ N. Chevalier, ${ }^{8}$ W. N. Cottingham, ${ }^{8}$ C. Mackay ${ }^{8}$ F. F. Wilson, ${ }^{8}$ C. Hearty,${ }^{9}$ T. S. Mattison,${ }^{9}$ J. A. McKenna,${ }^{9}$ D. Thiessen, ${ }^{9}$ P. Kyberd,${ }^{10}$ A. K. McKemey, ${ }^{10}$ V. E. Blinov, ${ }^{11}$ A. D. Bukin, ${ }^{11}$ V. B. Golubev, ${ }^{11}$ V. N. Ivanchenko, ${ }^{11}$ E. A. Kravchenko, ${ }^{11}$ A. P. Onuchin, ${ }^{11}$ S. I. Serednyakov,${ }^{11}$ Yu. I. Skovpen,${ }^{11}$ E. P. Solodov, ${ }^{11}$ A. N. Yushkov,${ }^{11}$ D. Best,${ }^{12}$ M. Chao, ${ }^{12}$ D. Kirkby ${ }^{12}$ A. J. Lankford, ${ }^{12}$ M. Mandelkern,${ }^{12}$ S. McMahon, ${ }^{12}$ R. K. Mommsen, ${ }^{12}$ W. Roethel, ${ }^{12}$ D. P. Stoker, ${ }^{12}$ C. Buchanan, ${ }^{13}$ H. K. Hadavand, ${ }^{14}$ E. J. Hill,,${ }^{14}$ D. B. MacFarlane, ${ }^{14}$ H. P. Paar, ${ }^{14}$ Sh. Rahatlou, ${ }^{14}$ U. Schwanke, ${ }^{14}$ V. Sharma, ${ }^{14}$ J. W. Berryhill, ${ }^{15}$ C. Campagnari, ${ }^{15}$ B. Dahmes,${ }^{15}$ N. Kuznetsova, ${ }^{15}$ S. L. Levy, ${ }^{15}$ O. Long, ${ }^{15}$ A. Lu ${ }^{15}$ M. A. Mazur, ${ }^{15}$ J. D. Richman, ${ }^{15}$ W. Verkerke, ${ }^{15}$ J. Beringer ${ }^{16}$ A. M. Eisner, ${ }^{16}$ C. A. Heusch,${ }^{16}$ W. S. Lockman, ${ }^{16}$ T. Schalk, ${ }^{16}$ R. E. Schmitz, ${ }^{16}$ B. A. Schumm, ${ }^{16}$ A. Seiden,${ }^{16}$ M. Turri,${ }^{16}$ W. Walkowiak,${ }^{16}$ D. C. Williams, ${ }^{16}$ M. G. Wilson, ${ }^{16}$ J. Albert, ${ }^{17}$ E. Chen, ${ }^{17}$ M. P. Dorsten,${ }^{17}$ G. P. Dubois-Felsmann, ${ }^{17}$ A. Dvoretskii, ${ }^{17}$ D. G. Hitlin, ${ }^{17}$ I. Narsky, ${ }^{17}$ F. C. Porter,${ }^{17}$ A. Ryd,${ }^{17}$ A. Samuel, ${ }^{17}$ S. Yang, ${ }^{17}$ S. Jayatilleke, ${ }^{18}$ G. Mancinelli, ${ }^{18}$ B. T. Meadows,${ }^{18}$ M. D. Sokoloff, ${ }^{18}$ T. Barillari, ${ }^{19}$ F. Blanc,${ }^{19}$ P. Bloom, ${ }^{19}$ P. J. Clark, ${ }^{19}$ W. T. Ford, ${ }^{19}$ U. Nauenberg,${ }^{19}$ A. Olivas,${ }^{19}$ P. Rankin, ${ }^{19}$ J. Roy ${ }^{19}$ J. G. Smith, ${ }^{19}$ W. C. van Hoek, ${ }^{19}$ L. Zhang, ${ }^{19}$ J. L. Harton, ${ }^{20}$ T. Hu, ${ }^{20}$ A. Soffer, ${ }^{20}$ W. H. Toki, ${ }^{20}$ R. J. Wilson, ${ }^{20}$ J. Zhang, ${ }^{20}$ D. Altenburg, ${ }^{21}$ T. Brandt,${ }^{21}$ J. Brose,${ }^{21}$ T. Colberg, ${ }^{21}$ M. Dickopp ${ }^{21}$ R. S. Dubitzky, ${ }^{21}$ A. Hauke,${ }^{21}$ H. M. Lacker, ${ }^{21}$ E. Maly, ${ }^{21}$ R. Müller-Pfefferkorn, ${ }^{21}$ R. Nogowski, ${ }^{21}$ S. Otto, ${ }^{21}$

K. R. Schubert ${ }^{21}$ R. Schwierz, ${ }^{21}$ B. Spaan, ${ }^{21}$ L. Wilden, ${ }^{21}$ D. Bernard,${ }^{22}$ G. R. Bonneaud,${ }^{22}$ F. Brochard, ${ }^{22}$

J. Cohen-Tanugi,${ }^{22}$ Ch. Thiebaux, ${ }^{22}$ G. Vasileiadis,${ }^{22}$ M. Verderi, ${ }^{22}$ A. Khan,${ }^{23}$ D. Lavin, ${ }^{23}$ F. Muheim, ${ }^{23}$

S. Playfer, ${ }^{23}$ J. E. Swain, ${ }^{23}$ J. Tinslay, ${ }^{23}$ C. Bozzi ${ }^{24}$ L. Piemontese,${ }^{24}$ A. Sarti,${ }^{24}$ E. Treadwell ${ }^{25}$ F. Anulli, ${ }^{26, *}$ R. Baldini-Ferroli, ${ }^{26}$ A. Calcaterra,${ }^{26}$ R. de Sangro, ${ }^{26}$ D. Falciai, ${ }^{26}$ G. Finocchiaro, ${ }^{26}$ P. Patteri, ${ }^{26}$ I. M. Peruzzi,${ }^{26, *}$ M. Piccolo ${ }^{26}$ A. Zallo, ${ }^{26}$ A. Buzzo,${ }^{27}$ R. Contri, ${ }^{27}$ G. Crosetti,${ }^{27}$ M. Lo Vetere,${ }^{27}$ M. Macri, ${ }^{27}$ M. R. Monge, ${ }^{27}$ S. Passaggio, ${ }^{27}$ F. C. Pastore, ${ }^{27}$ C. Patrignani, ${ }^{27}$ E. Robutti, ${ }^{27}$ A. Santroni, ${ }^{27}$ S. Tosi, ${ }^{27}$ S. Bailey, ${ }^{28}$ M. Morii, ${ }^{28}$ G. J. Grenier, ${ }^{29}$ S.-J. Lee, ${ }^{29}$ U. Mallik,${ }^{29}$ J. Cochran, ${ }^{30}$ H. B. Crawley ${ }^{30}$ J. Lamsa, ${ }^{30}$ W. T. Meyer,${ }^{30}$ S. Prell, ${ }^{30}$

E. I. Rosenberg, ${ }^{30}$ J. Yi, ${ }^{30}$ M. Davier,${ }^{31}$ G. Grosdidier ${ }^{31}$ A. Höcker, ${ }^{31}$ S. Laplace,${ }^{31}$ F. Le Diberder,${ }^{31}$ V. Lepeltier, ${ }^{31}$

A. M. Lutz,${ }^{31}$ T. C. Petersen, ${ }^{31}$ S. Plaszczynski, ${ }^{31}$ M. H. Schune, ${ }^{31}$ L. Tantot,${ }^{31}$ G. Wormser,${ }^{31}$ R. M. Bionta, ${ }^{32}$ V. Brigljević, ${ }^{32}$ C. H. Cheng, ${ }^{32}$ D. J. Lange, ${ }^{32}$ D. M. Wright,${ }^{32}$ A. J. Bevan,${ }^{33}$ J. R. Fry,${ }^{33}$ E. Gabathuler ${ }^{33}$ R. Gamet, ${ }^{33}$ M. Kay, ${ }^{33}$ D. J. Payne, ${ }^{33}$ R. J. Sloane, ${ }^{33}$ C. Touramanis, ${ }^{33}$ M. L. Aspinwall, ${ }^{34}$ D. A. Bowerman,${ }^{34}$ P. D. Dauncey,$^{34}$ U. Egede, ${ }^{34}$ I. Eschrich, ${ }^{34}$ G. W. Morton,${ }^{34}$ J. A. Nash,${ }^{34}$ P. Sanders,${ }^{34}$ G. P. Taylor,${ }^{34}$ J. J. Back ${ }^{35}$ G. Bellodi ${ }^{35}$ P. F. Harrison ${ }^{35}$ H. W. Shorthouse, ${ }^{35}$ P. Strother, ${ }^{35}$ P. B. Vidal,,${ }^{35}$ G. Cowan, ${ }^{36}$ H. U. Flaecher, ${ }^{36}$ S. George ${ }^{36}$ M. G. Green, ${ }^{36}$ A. Kurup,${ }^{36}$ C. E. Marker, ${ }^{36}$ T. R. McMahon, ${ }^{36}$ S. Ricciardi, ${ }^{36}$ F. Salvatore, ${ }^{36}$ G. Vaitsas, ${ }^{36}$ M. A. Winter, ${ }^{36}$ D. Brown, ${ }^{37}$ C. L. Davis, ${ }^{37}$ J. Allison, ${ }^{38}$ R. J. Barlow, ${ }^{38}$ A. C. Forti, ${ }^{38}$ P. A. Hart, ${ }^{38}$ F. Jackson, ${ }^{38}$ G. D. Lafferty, ${ }^{38}$ A. J. Lyon, ${ }^{38}$ J. H. Weatherall, ${ }^{38}$ J. C. Williams ${ }^{38}$ A. Farbin, ${ }^{39}$ A. Jawahery, ${ }^{39}$ D. Kovalskyi, ${ }^{39}$ C. K. Lae,${ }^{39}$ V. Lillard, ${ }^{39}$ D. A. Roberts, ${ }^{39}$ G. Blaylock, ${ }^{40}$ C. Dallapiccola,${ }^{40}$ K. T. Flood, ${ }^{40}$ S. S. Hertzbach, ${ }^{40}$ R. Kofler, ${ }^{40}$ V. B. Koptchev ${ }^{40}$ T. B. Moore,${ }^{40}$ H. Staengle,${ }^{40}$ S. Willocq, ${ }^{40}$ R. Cowan, ${ }^{41}$ G. Sciolla, ${ }^{41}$ F. Taylor,${ }^{41}$ R. K. Yamamoto, ${ }^{41}$ D. J. J. Mangeol, ${ }^{42}$ M. Milek, ${ }^{42}$ P. M. Patel, ${ }^{42}$ A. Lazzaro, ${ }^{43}$ F. Palombo, ${ }^{43}$ J. M. Bauer ${ }^{44}$ L. Cremaldi, ${ }^{44}$ V. Eschenburg, ${ }^{44}$ R. Godang, ${ }^{44}$ R. Kroeger ${ }^{44}$ J. Reidy ${ }^{44}$ D. A. Sanders, ${ }^{44}$ D. J. Summers, ${ }^{44}$ H. W. Zhao, ${ }^{44}$ C. Hast,,${ }^{45}$ P. Taras,${ }^{45}$ H. Nicholson, ${ }^{46}$ C. Cartaro,${ }^{47}$ N. Cavallo ${ }^{47}$ G. De Nardo,${ }^{47}$ F. Fabozzi $,{ }^{47}, \dagger$ C. Gatto ${ }^{47}$ L. Lista, ${ }^{47}$ P. Paolucci, ${ }^{47}$ D. Piccolo,${ }^{47}$ C. Sciacca, ${ }^{47}$ M. A. Baak ${ }^{48}$ G. Raven, ${ }^{48}$ J. M. LoSecco, ${ }^{49}$ T. A. Gabriel,${ }^{50}$ B. Brau,${ }^{51}$ T. Pulliam, ${ }^{51}$ J. Brau, ${ }^{52}$ R. Frey, ${ }^{52}$ M. Iwasaki,${ }^{52}$ 
C. T. Potter, ${ }^{52}$ N. B. Sinev, ${ }^{52}$ D. Strom, ${ }^{52}$ E. Torrence,${ }^{52}$ F. Colecchia, ${ }^{53}$ A. Dorigo, ${ }^{53}$ F. Galeazzi,${ }^{53}$ M. Margoni, ${ }^{53}$ M. Morandin, ${ }^{53}$ M. Posocco, ${ }^{53}$ M. Rotondo, ${ }^{53}$ F. Simonetto, ${ }^{53}$ R. Stroili, ${ }^{53}$ G. Tiozzo, ${ }^{53}$ C. Voci, ${ }^{53}$ M. Benayoun, ${ }^{54}$ H. Briand, ${ }^{54}$ J. Chauveau, ${ }^{54}$ P. David ${ }^{54}$ Ch. de la Vaissière,${ }^{54}$ L. Del Buono, ${ }^{54}$ O. Hamon, ${ }^{54}$ Ph. Leruste,${ }^{54}$ J. Ocariz,${ }^{54}$ M. Pivk,${ }^{54}$ L. Roos,${ }^{54}$ J. Stark,${ }^{54}$ S. T'Jampens, ${ }^{54}$ P. F. Manfredi, ${ }^{55}$ V. Re,${ }^{55}$ L. Gladney, ${ }^{56}$ Q. H. Guo, ${ }^{56}$ J. Panetta, ${ }^{56}$ C. Angelini, ${ }^{57}$ G. Batignani, ${ }^{57}$ S. Bettarini,${ }^{57}$ M. Bondioli, ${ }^{57}$ F. Bucci, ${ }^{57}$ G. Calderini,${ }^{57}$ M. Carpinelli, ${ }^{57}$ F. Forti, ${ }^{57}$ M. A. Giorgi, ${ }^{57}$ A. Lusiani, ${ }^{57}$ G. Marchiori, ${ }^{57}$ F. Martinez-Vidal,,${ }^{57}$ ‡ M. Morganti, ${ }^{57}$ N. Neri, ${ }^{57}$ E. Paoloni,${ }^{57}$ M. Rama,${ }^{57}$ G. Rizzo,${ }^{57}$ F. Sandrelli, ${ }^{57}$ J. Walsh,${ }^{57}$ M. Haire,${ }^{58}$ D. Judd ${ }^{58}$ K. Paick, ${ }^{58}$ D. E. Wagoner ${ }^{58}$ N. Danielson, ${ }^{59}$ P. Elmer, ${ }^{59}$ C. Lu,${ }^{59}$ V. Miftakov, ${ }^{59}$ J. Olsen, ${ }^{59}$ A. J. S. Smith, ${ }^{59}$ E. W. Varnes, ${ }^{59}$ F. Bellini, ${ }^{60}$ G. Cavoto,${ }^{59,60}$ D. del Re,${ }^{60}$ R. Faccini, ${ }^{14,60}$ F. Ferrarotto,${ }^{60}$ F. Ferroni, ${ }^{60}$ M. Gaspero, ${ }^{60}$ E. Leonardi,${ }^{60}$ M. A. Mazzoni, ${ }^{60}$ S. Morganti, ${ }^{60}$ M. Pierini, ${ }^{60}$ G. Piredda, ${ }^{60}$ F. Safai Tehrani,${ }^{60}$ M. Serra,${ }^{60}$ C. Voena,${ }^{60}$ S. Christ,${ }^{61}$ G. Wagner, ${ }^{61}$ R. Waldi,${ }^{61}$ T. Adye,${ }^{62}$ N. De Groot,${ }^{62}$ B. Franek,${ }^{62}$ N. I. Geddes,${ }^{62}$ G. P. Gopal, ${ }^{62}$ E. O. Olaiya, ${ }^{62}$ S. M. Xella, ${ }^{62}$ R. Aleksan, ${ }^{63}$ S. Emery, ${ }^{63}$ A. Gaidot ${ }^{63}$ S. F. Ganzhur, ${ }^{63}$ P.-F. Giraud ${ }^{63}$ G. Hamel de Monchenault ${ }^{63}$ W. Kozanecki, ${ }^{63}$ M. Langer, ${ }^{63}$ G. W. London, ${ }^{63}$ B. Mayer, ${ }^{63}$ G. Schott,${ }^{63}$ G. Vasseur, ${ }^{63}$ Ch. Yeche,${ }^{63}$ M. Zito, ${ }^{63}$ M. V. Purohit ${ }^{64}$ A. W. Weidemann, ${ }^{64}$ F. X. Yumiceva, ${ }^{64}$ D. Aston,${ }^{65}$ R. Bartoldus, ${ }^{65}$ N. Berger,${ }^{65}$ A. M. Boyarski, ${ }^{65}$ O. L. Buchmueller ${ }^{65}$ M. R. Convery ${ }^{65}$ D. P. Coupal, ${ }^{65}$ D. Dong, ${ }^{65}$ J. Dorfan, ${ }^{65}$ D. Dujmic,${ }^{65}$ W. Dunwoodie ${ }^{65}$ R. C. Field,${ }^{65}$ T. Glanzman,${ }^{65}$ S. J. Gowdy, ${ }^{65}$ E. Grauges-Pous, ${ }^{65}$ T. Hadig, ${ }^{65}$ V. Halyo, ${ }^{65}$ T. Hryn'ova, ${ }^{65}$ W. R. Innes ${ }^{65}$ C. P. Jessop,${ }^{65}$ M. H. Kelsey, ${ }^{65}$ P. Kim,${ }^{65}$ M. L. Kocian,${ }^{65}$ U. Langenegger ${ }^{65}$ D. W. G. S. Leith, ${ }^{65}$ S. Luitz, ${ }^{65}$ V. Luth,${ }^{65}$ H. L. Lynch,${ }^{65}$ H. Marsiske, ${ }^{65}$ S. Menke, ${ }^{65}$ R. Messner ${ }^{65}$ D. R. Muller ${ }^{65}$ C. P. O'Grady, ${ }^{65}$ V. E. Ozcan, ${ }^{65}$ A. Perazzo,${ }^{65}$ M. Perl, ${ }^{65}$ S. Petrak, ${ }^{65}$ B. N. Ratcliff, ${ }^{65}$ S. H. Robertson, ${ }^{65}$ A. Roodman ${ }^{65}$ A. A. Salnikov ${ }^{65}$ R. H. Schindler, ${ }^{65}$ J. Schwiening, ${ }^{65}$ G. Simi, ${ }^{65}$ A. Snyder, ${ }^{65}$ A. Soha, ${ }^{65}$ J. Stelzer, ${ }^{65}$ D. Su, ${ }^{65}$ M. K. Sullivan,${ }^{65}$ H. A. Tanaka, ${ }^{65}$ J. Va'vra ${ }^{65}$ S. R. Wagner,${ }^{65}$ M. Weaver,${ }^{65}$ A. J. R. Weinstein, ${ }^{65}$ W. J. Wisniewski, ${ }^{65}$ D. H. Wright,${ }^{65}$ C. C. Young, ${ }^{65}$ P. R. Burchat, ${ }^{66}$ T. I. Meyer, ${ }^{66}$ C. Roat, ${ }^{66}$ S. Ahmed, ${ }^{67}$ J. A. Ernst,${ }^{67}$ W. Bugg, ${ }^{68}$ M. Krishnamurthy, ${ }^{68}$ S. M. Spanier,${ }^{68}$ R. Eckmann,${ }^{69}$ H. Kim, ${ }^{69}$ J. L. Ritchie, ${ }^{69}$ R. F. Schwitters,${ }^{69}$ J. M. Izen,${ }^{70}$ I. Kitayama, ${ }^{70}$ X. C. Lou, ${ }^{70}$ S. Ye,${ }^{70}$ F. Bianchi, ${ }^{71}$ M. Bona,${ }^{71}$ F. Gallo, ${ }^{71}$ D. Gamba,${ }^{71}$ C. Borean,${ }^{72}$ L. Bosisio, ${ }^{72}$ G. Della Ricca,${ }^{72}$ S. Dittongo,${ }^{72}$ S. Grancagnolo,${ }^{72}$ L. Lanceri, ${ }^{72}$ P. Poropat, ${ }^{72}, \S$ L. Vitale, ${ }^{72}$ G. Vuagnin,,${ }^{72}$ R. S. Panvini, ${ }^{73}$ Sw. Banerjee,${ }^{74}$ C. M. Brown, ${ }^{74}$ D. Fortin, ${ }^{74}$ P. D. Jackson, ${ }^{74}$ R. Kowalewski, ${ }^{74}$ J. M. Roney, ${ }^{74}$ H. R. Band, ${ }^{75}$ S. Dasu, ${ }^{75}$ M. Datta, ${ }^{75}$ A. M. Eichenbaum, ${ }^{75}$ H. Hu ${ }^{75}$ J. R. Johnson, ${ }^{75}$ R. Liu, ${ }^{75}$ F. Di Lodovico, ${ }^{75}$ A. K. Mohapatra, ${ }^{75}$ Y. Pan, ${ }^{75}$ R. Prepost, ${ }^{75}$ S. J. Sekula, ${ }^{75}$ J. H. von Wimmersperg-Toeller, ${ }^{75}$ J. Wu ${ }^{75}$ S. L. Wu ${ }^{75}$ Z. Yu, ${ }^{75}$ and H. Neal ${ }^{76}$

(The BABAR Collaboration)

\author{
${ }^{1}$ Laboratoire de Physique des Particules, F-74941 Annecy-le-Vieux, France \\ ${ }^{2}$ Università di Bari, Dipartimento di Fisica and INFN, I-70126 Bari, Italy \\ ${ }^{3}$ Institute of High Energy Physics, Beijing 100039, China \\ ${ }^{4}$ University of Bergen, Inst. of Physics, N-5007 Bergen, Norway \\ ${ }^{5}$ Lawrence Berkeley National Laboratory and University of California, Berkeley, CA 94720, USA \\ ${ }^{6}$ University of Birmingham, Birmingham, B15 2TT, United Kingdom \\ ${ }^{7}$ Ruhr Universität Bochum, Institut für Experimentalphysik 1, D-44780 Bochum, Germany \\ ${ }^{8}$ University of Bristol, Bristol BS8 1TL, United Kingdom \\ ${ }^{9}$ University of British Columbia, Vancouver, BC, Canada V6T $1 Z 1$ \\ ${ }^{10}$ Brunel University, Uxbridge, Middlesex UB8 3PH, United Kingdom \\ ${ }^{11}$ Budker Institute of Nuclear Physics, Novosibirsk 630090, Russia \\ ${ }^{12}$ University of California at Irvine, Irvine, CA 92697, USA \\ ${ }^{13}$ University of California at Los Angeles, Los Angeles, CA 90024, USA \\ ${ }^{14}$ University of California at San Diego, La Jolla, CA 92093, USA \\ ${ }^{15}$ University of California at Santa Barbara, Santa Barbara, CA 93106, USA \\ ${ }^{16}$ University of California at Santa Cruz, Institute for Particle Physics, Santa Cruz, CA 95064, USA \\ ${ }^{17}$ California Institute of Technology, Pasadena, CA 91125, USA \\ ${ }^{18}$ University of Cincinnati, Cincinnati, $O H$ 45221, USA \\ ${ }^{19}$ University of Colorado, Boulder, CO 80309, USA \\ ${ }^{20}$ Colorado State University, Fort Collins, CO 80523, USA \\ ${ }^{21}$ Technische Universität Dresden, Institut für Kern- und Teilchenphysik, D-01062 Dresden, Germany \\ ${ }^{22}$ Ecole Polytechnique, LLR, F-91128 Palaiseau, France \\ ${ }^{23}$ University of Edinburgh, Edinburgh EH9 3JZ, United Kingdom \\ ${ }^{24}$ Università di Ferrara, Dipartimento di Fisica and INFN, I-44100 Ferrara, Italy \\ ${ }^{25}$ Florida A $8 M$ University, Tallahassee, FL 32307, USA \\ ${ }^{26}$ Laboratori Nazionali di Frascati dell'INFN, I-00044 Frascati, Italy
}


${ }^{27}$ Università di Genova, Dipartimento di Fisica and INFN, I-16146 Genova, Italy

${ }^{28}$ Harvard University, Cambridge, MA 02138, USA

${ }^{29}$ University of Iowa, Iowa City, IA 52242, USA

${ }^{30}$ Iowa State University, Ames, IA 50011-3160, USA

${ }^{31}$ Laboratoire de l'Accélérateur Linéaire, F-91898 Orsay, France

${ }^{32}$ Lawrence Livermore National Laboratory, Livermore, CA 94550, USA

${ }^{33}$ University of Liverpool, Liverpool L69 3BX, United Kingdom

${ }^{34}$ University of London, Imperial College, London, SW7 2BW, United Kingdom

${ }^{35}$ Queen Mary, University of London, E1 4NS, United Kingdom

${ }^{36}$ University of London, Royal Holloway and Bedford New College, Egham, Surrey TW20 0EX, United Kingdom

${ }^{37}$ University of Louisville, Louisville, KY 40292, USA

${ }^{38}$ University of Manchester, Manchester M13 9PL, United Kingdom

${ }^{39}$ University of Maryland, College Park, MD 20742, USA

${ }^{40}$ University of Massachusetts, Amherst, MA 01003, USA

${ }^{41}$ Massachusetts Institute of Technology, Laboratory for Nuclear Science, Cambridge, MA 02139, USA

${ }^{42}$ Mc Gill University, Montréal, QC, Canada H3A $2 T 8$

${ }^{43}$ Università di Milano, Dipartimento di Fisica and INFN, I-20133 Milano, Italy

${ }^{44}$ University of Mississippi, University, MS 38677, USA

${ }^{45}$ Université de Montréal, Laboratoire René J. A. Lévesque, Montréal, QC, Canada H3C 3J7

${ }_{46}^{6}$ Mount Holyoke College, South Hadley, MA 01075, USA

${ }^{47}$ Università di Napoli Federico II, Dipartimento di Scienze Fisiche and INFN, I-80126, Napoli, Italy

${ }^{48}$ NIKHEF, National Institute for Nuclear Physics and High Energy Physics, 1009 DB Amsterdam, The Netherlands

${ }^{49}$ University of Notre Dame, Notre Dame, IN 46556, USA

${ }^{50}$ Oak Ridge National Laboratory, Oak Ridge, TN 37831, USA

${ }^{51}$ Ohio State University, Columbus, OH 43210, USA

${ }^{52}$ University of Oregon, Eugene, OR 97403, USA

${ }^{53}$ Università di Padova, Dipartimento di Fisica and INFN, I-35131 Padova, Italy

${ }^{54}$ Universités Paris VI et VII, Lab de Physique Nucléaire H. E., F-75252 Paris, France

${ }^{55}$ Università di Pavia, Dipartimento di Elettronica and INFN, I-27100 Pavia, Italy

${ }^{56}$ University of Pennsylvania, Philadelphia, PA 19104, USA

${ }^{57}$ Università di Pisa, Dipartimento di Fisica, Scuola Normale Superiore and INFN, I-56127 Pisa, Italy

${ }^{58}$ Prairie View A\&M University, Prairie View, TX 77446, USA

${ }^{59}$ Princeton University, Princeton, NJ 08544, USA

${ }^{60}$ Università di Roma La Sapienza, Dipartimento di Fisica and INFN, I-00185 Roma, Italy

${ }^{61}$ Universität Rostock, D-18051 Rostock, Germany

${ }^{62}$ Rutherford Appleton Laboratory, Chilton, Didcot, Oxon, OX11 OQX, United Kingdom

${ }^{63}$ DAPNIA, Commissariat à l'Energie Atomique/Saclay, F-91191 Gif-sur-Yvette, France

${ }^{64}$ University of South Carolina, Columbia, SC 29208, USA

${ }^{65}$ Stanford Linear Accelerator Center, Stanford, CA 94309, USA

${ }^{66}$ Stanford University, Stanford, CA 94305-4060, USA

${ }^{67}$ State Univ. of New York, Albany, NY 12222, USA

${ }^{68}$ University of Tennessee, Knoxville, TN 37996, USA

${ }^{69}$ University of Texas at Austin, Austin, TX 78712, USA

${ }^{70}$ University of Texas at Dallas, Richardson, TX 75083, USA

${ }^{71}$ Università di Torino, Dipartimento di Fisica Sperimentale and INFN, I-10125 Torino, Italy

${ }^{72}$ Università di Trieste, Dipartimento di Fisica and INFN, I-34127 Trieste, Italy

${ }^{73}$ Vanderbilt University, Nashville, TN 37235, USA

${ }^{74}$ University of Victoria, Victoria, BC, Canada V8W $3 P 6$

${ }^{75}$ University of Wisconsin, Madison, WI 53706, USA

${ }^{76}$ Yale University, New Haven, CT 06511, USA

(Dated: March 24, 2003)

We have performed a search for the decays $B^{+} \rightarrow J / \psi p \bar{\Lambda}$ and $B^{0} \rightarrow J / \psi p \bar{p}$ in a data set of $(88.9 \pm$ $1.0) \times 10^{6} \Upsilon(4 S)$ decays collected by the BABAR experiment at the PEP-II $e^{+} e^{-}$storage ring at the Stanford Linear Accelerator Center. Four charged $B$ candidates have been observed with an expected background of $0.21 \pm 0.14$ events. The corresponding branching fraction is $\left(12_{-6}^{+9}\right) \times 10^{-6}$, where statistical and systematic uncertainties have been combined. The result can be interpreted as a $90 \%$ confidence level (CL) upper limit of $26 \times 10^{-6}$. We also find one $B^{0}$ candidate, with an expected background of $0.64 \pm 0.17$ events, implying a $90 \%$ CL upper limit of $1.9 \times 10^{-6}$.

PACS numbers: 13.20.He, 12.39.Mk, 12.39.Jh

Studies of the inclusive production of charmonium

mesons in $B$ decays at the $\Upsilon(4 S)$ resonance have been 


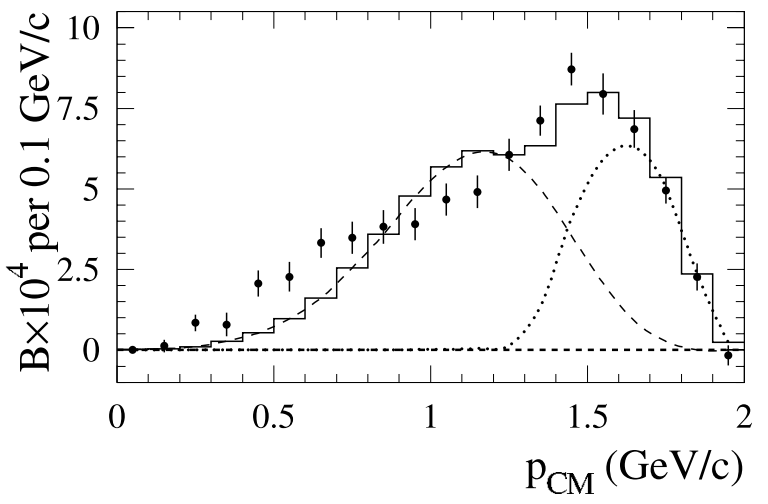

FIG. 1: Center-of-mass momentum of $J / \psi$ mesons produced directly in $B$ decays (points). The histogram is the sum of the color-octet component from a recent NRQCD calculation [4] (dashed line), which includes multi-body final states, and the color-singlet $J / \psi K^{(*)}$ component from simulation [5] (dotted line).

published by CLEO [1] and BABAR [2], and preliminary results have been presented by Belle [3]. One of the interesting features observed by all three collaborations is an excess of $J / \psi$ mesons at low momentum in the $e^{+} e^{-}$ center-of-mass frame, $p_{C M}$, when compared to distributions predicted by non-relativistic QCD calculations [4]. Figure 1 (from Ref. [2]) shows $p_{C M}$ for $J / \psi$ mesons produced in $B$ decay after subtraction of the component due to the decay of heavier charmonium states. The excess below $0.8 \mathrm{GeV} / c$ corresponds to a branching fraction of approximately $6 \times 10^{-4}, 8 \%$ of the total direct $J / \psi$ production.

Possible sources of the excess include an intrinsic charm component of the $B[6]$ or the production of an $s \bar{d} g$ hybrid [7] in conjunction with a $J / \psi$. Another possibility [8] is that the excess is from decays of the form $B \rightarrow J / \psi$ baryon anti-baryon. The rate of these decays could be enhanced by the intermediate production of an exotic state allowed by QCD but not yet observed, including nuclear-bound quarkonium (a $c \bar{c}$ pair bound to a nucleon), baryonium (a baryon-antibaryon bound state), or a pentaquark (a baryon containing five quarks). If such resonances were narrow, the other particle in the decay would be monoenergetic in the $B$ rest frame. Note that the $J / \psi$ spectrum in Fig. 1 would not directly display such narrow distributions because it is measured in the $e^{+} e^{-}$center-of-mass frame. The difference between $p_{C M}$ and $p^{*}$, the $J / \psi$ momentum in the $B$ rest frame, has an RMS of $0.12 \mathrm{GeV} / c$ due to the motion of the $B$.

This Letter presents searches for the decays $B^{+} \rightarrow J / \psi p \bar{\Lambda}$ and $B^{0} \rightarrow J / \psi p \bar{p}$ in a sample of $81.9 \mathrm{fb}^{-1}$ collected by the BABAR detector. Note that the latter decay is Cabibbo suppressed relative to the former. Charge conjugation is implied throughout.

BABAR operates at the PEP-II $e^{+} e^{-}$storage ring, which collides $9.0 \mathrm{GeV}$ electrons on $3.1 \mathrm{GeV}$ positrons to create a center-of-mass system with energy $10.58 \mathrm{GeV}$ moving along the $z$ axis with a Lorentz boost of $\beta \gamma=$ 0.55. $\Upsilon(4 S)$ production makes up approximately $23 \%$ of the total hadronic cross section.

The BABAR detector is described in detail in Ref. [9]. The trajectories of charged particles are reconstructed and their momenta measured with two detector systems located in a 1.5-T solenoidal magnetic field: a five-layer, double-sided silicon vertex tracker (SVT) and a 40-layer drift chamber $(\mathrm{DCH})$. The tracking fiducial volume covers the polar angular region $0.41<\theta<2.54 \mathrm{rad}$, which is $86 \%$ of the solid angle in the center-of-mass frame. The transverse momentum resolution is $0.49 \%$ at $0.3 \mathrm{GeV} / c$ and $0.59 \%$ at $1 \mathrm{GeV} / c$.

The energies deposited by charged tracks and photons are measured by a $\mathrm{CsI}(\mathrm{Tl})$ calorimeter $(\mathrm{EMC})$ in the fiducial volume $0.41<\theta<2.41 \mathrm{rad}$ ( $84 \%$ of the centerof-mass solid angle) with energy resolution at $1 \mathrm{GeV}$ of $2.6 \%$. Muons are detected in the IFR, a multilayer device of resistive plate chambers located in the flux return of the solenoid. The DIRC, a Cherenkov radiation detector, is used to identify charged particles.

We select $B$ candidates of interest in a $B \bar{B}$-enriched sample. Events in the sample are required to have visible energy $E$ greater than $4.5 \mathrm{GeV}$ and a ratio of the second to the zeroth Fox-Wolfram moment [10], $R_{2}$, less than 0.5. Both $E$ and $R_{2}$ are calculated from tracks and neutral energy deposits in the respective fiducial volumes noted above. The same tracks are used to construct a primary event vertex, which is required to be located within $6 \mathrm{~cm}$ of the beam spot in $z$ and within $0.5 \mathrm{~cm}$ of the beam line. The beam spot RMS size is approximately $0.9 \mathrm{~cm}$ in $z, 120 \mu \mathrm{m}$ horizontally, and $5.6 \mu \mathrm{m}$ vertically.

There must be at least three tracks in the fiducial volume satisfying the following quality criteria: they must have transverse momentum greater than $0.1 \mathrm{GeV} / c$, momentum less than $10 \mathrm{GeV} / c$, at least 12 hits in the $\mathrm{DCH}$, and approach within $10 \mathrm{~cm}$ of the beam spot in $z$ and within $1.5 \mathrm{~cm}$ of the beam line.

Studies with simulated data indicate that these criteria are satisfied by $96 \%$ of generic $B \bar{B}$ events.

$B^{+} \rightarrow J / \psi p \bar{\Lambda}$ candidates are formed by combining $J / \psi$, proton, and $\bar{\Lambda}$ candidates. $J / \psi$ candidates must have mass in the range $2.950-3.130 \mathrm{GeV} / c^{2}$ if reconstructed in the $e^{+} e^{-}$final state or $3.060-3.130 \mathrm{GeV} / c^{2}$ in $\mu^{+} \mu^{-}$.

One of the two electrons from the $J / \psi$ must satisfy the following ("tight") requirements. It must have an energy deposit in the EMC between $89 \%$ and $120 \%$ of its momentum, a Cherenkov angle in the DIRC within $3 \sigma$ of expectation for an electron, a lateral moment of the energy deposit [11], LAT, between 0.1 and 0.6 , an $A_{42}$ Zernike moment [12] less than 0.11, and an energy loss in the DCH consistent with expectation. Less stringent ("loose") requirements are imposed in the selection of the second electron: we require an energy deposit in 
the EMC of at least $65 \%$ of its momentum and place a less restrictive requirement on DCH energy, with no requirements on LAT or $A_{42}$. Whenever possible, photons radiated by an electron traversing material prior to the DCH (0.04 radiation lengths at normal incidence) are combined with the track [2].

At $1.5 \mathrm{GeV} / c$, a typical lepton momentum, the tighter criteria have an efficiency of $91 \%$ with a pion misidentification probability of $0.13 \%$. The looser criteria give $98 \%$ efficiency with $3 \%$ pion misidentification.

Muon candidates must deposit less than $0.5 \mathrm{GeV}$ in the EMC (2.3 times the minimum-ionizing peak) and have a pattern of hits in the IFR consistent with the trajectory of a muon. The total amount of material penetrated must be greater than 2 interaction lengths and must be within 2 interaction lengths of the value expected for a muon. The muon identification efficiency at $1.5 \mathrm{GeV} / c$ is $77 \%$ with a pion misidentification probability of $11 \%$.

Proton candidates are selected with a likelihood method that uses the energy deposited in the SVT and the DCH, and the Cherenkov angle and number of photons observed in the DIRC. They are also required to fail the tight electron identification criteria. At a typical momentum of $300 \mathrm{MeV} / c$, the selection efficiency is greater than $98 \%$ with a kaon misidentification probability less than $1 \%$.

The $\bar{\Lambda}$ is reconstructed from a proton, which must satisfy the above criteria, and an oppositely charged track, assumed to be a pion. It must have mass between 1.10 and $1.14 \mathrm{GeV} / c^{2}$, and a vertex that is separated from the $J / \psi$ vertex by at least $2 \mathrm{~mm}$. The angle between the $\bar{\Lambda}$ momentum and the vector from the $J / \psi$ vertex to the $\bar{\Lambda}$ vertex must be less than $90^{\circ}$ in the laboratory frame.

Geometrical vertex fits are performed on the resulting $B^{+}$candidates, of which approximately $68 \%$ are rejected by a requirement on the quality of the fit.

$B^{0} \rightarrow J / \psi p \bar{p}$ candidates are formed from $J / \psi$ candidates and an oppositely-charged pair of proton candidates. Approximately $83 \%$ of resulting candidates fail a requirement on the quality of a vertex fit.

We use two nearly-independent kinematic variables [9] to categorize $B$ candidates: the difference between the reconstructed and expected energy of the $B$ candidate in the $e^{+} e^{-}$center-of-mass frame, $\Delta E=\left(q_{\Upsilon} \cdot q_{B}-\right.$ $s / 2) / \sqrt{s}$, and the beam-energy substituted mass, $m_{\mathrm{ES}}=$ $\sqrt{\left(0.5 s+\vec{p}_{B} \cdot \vec{p}_{\Upsilon}\right)^{2} / E_{\Upsilon}^{2}-p_{B}^{2}}$. The four-momentum of the $e^{+} e^{-}$initial state, obtained from the beam momenta, is $q_{\Upsilon}=\left(E_{\Upsilon}, \vec{p}_{\Upsilon}\right)$, and $s \equiv\left|q_{\Upsilon}\right|^{2}$. The four-momentum of the reconstructed $B$ candidate, $q_{B}=\left(E_{B}, \vec{p}_{B}\right)$, is found by summing the four-momenta of the three daughters, with daughter masses constrained to accepted values [13].

The "analysis window" AW is defined by $5.2<m_{\mathrm{ES}}<$ $5.3 \mathrm{GeV} / c^{2}$ and $-0.10<\Delta E<0.25 \mathrm{GeV}$ ( $B^{+}$candidates) and $-0.25<\Delta E<0.25 \mathrm{GeV}$ ( $B^{0}$ candidates). The $\Delta E$ range is smaller for the charged candidates due to a kinematic cutoff in the $B^{+} \rightarrow J / \psi p \bar{\Lambda}$ decay. Only candidates in the AW are considered in the analysis. Approximately $15 \%$ of $B^{+}$events and $1.5 \%$ of $B^{0}$ events contain more than one candidate, in which case we select the one with the lowest $|\Delta E|$.

For signal events, $\langle\Delta E\rangle \approx 0$ and $\left\langle m_{\mathrm{ES}}\right\rangle \approx M_{B}$. We define a signal ellipse by $\left[\left(m_{\mathrm{ES}}-M_{B}\right) / \sigma_{m}\right]^{2}+\left[\Delta E / \sigma_{E}\right]^{2}<$ $S^{2}$, where the resolutions $\sigma_{m}$ and $\sigma_{E}$ are estimated from simulated data to be $3.1 \mathrm{MeV} / c^{2}$ and $6.5 \mathrm{MeV}$, respectively, for $B^{+} \rightarrow J / \psi p \bar{\Lambda}$, and $2.7 \mathrm{MeV} / c^{2}$ and $5.5 \mathrm{MeV}$ for $B^{0} \rightarrow J / \psi p \bar{p} . S=2.4$ for $B^{+} \rightarrow J / \psi p \bar{\Lambda}$ and $S=2.2$ for $B^{0} \rightarrow J / \psi p \bar{p}$.

The selection criteria for charged and neutral $B$ candidates, including the values for $S$, have been chosen to minimize the $90 \%$ CL upper limit expected in the absence of real signal, based on simulated signal and background events. Approximately $90 \%$ of the background events satisfying the criteria are combinatorial $B \bar{B}$, in which tracks from the decays of both $B$ mesons are used to form the candidate. The rest are continuum (non- $B \bar{B}$ ) events. Both components are distributed throughout the $\mathrm{AW}$, and neither peaks in the the signal of either $\Delta E$ or $m_{\mathrm{ES}}$.

We use simulated $B^{+} \rightarrow J / \psi p \bar{\Lambda}$ and $B^{0} \rightarrow J / \psi p \bar{p}$ events to measure the selection efficiency. The simulation does not include exotic QCD bound states. We study the accuracy of the simulation of the detector response by comparing data and simulated background events in samples similar to the final selection. We compare the number of $J / \psi$ mesons reconstructed in $B^{0} \rightarrow J / \psi p \bar{p}$ candidates in which only one proton satisfies the identification criteria, and we compare the number of $\bar{\Lambda}$ baryons reconstructed in $B^{+} \rightarrow J / \psi p \bar{\Lambda}$ candidates in which the proton daughter of the $B^{+}$is required to fail the criteria. Based on these studies, we apply multiplicative corrections to the efficiency of $0.97 \pm 0.06$ for $J / \psi$ reconstruction and $0.86 \pm 0.14$ for $\bar{\Lambda}$ reconstruction. We also compare the distributions of the $\chi^{2}$ of the $B$ vertex for candidates satisfying all other criteria and obtain corrections of $0.98 \pm 0.02$ for $B^{+} \rightarrow J / \psi p \bar{\Lambda}$ and $0.90 \pm 0.10$ for $B^{0} \rightarrow J / \psi p \bar{p}$.

The efficiency for $B^{+} \rightarrow J / \psi p \bar{\Lambda}$, with the $J / \psi$ decaying to $e^{+} e^{-}$or $\mu^{+} \mu^{-}$and $\bar{\Lambda}$ decaying to $\bar{p} \pi^{+}$, is $0.049 \pm 0.009$. The $18 \%$ fractional uncertainty includes $16 \%$ from $\bar{\Lambda}$ reconstruction, $6 \%$ from the $J / \psi, 3 \%$ from statistical uncertainty in the simulation, $2 \%$ from the $\chi^{2}$ correction, and $1 \%$ uncertainty on proton reconstruction efficiency. Approximately $25 \%$ of signal events satisfying all other criteria are reconstructed outside the signal ellipse.

The efficiency for $B^{0} \rightarrow J / \psi p \bar{p}$ with the $J / \psi$ decaying to $e^{+} e^{-}$or $\mu^{+} \mu^{-}$is $0.184 \pm 0.024$. The $13 \%$ uncertainty includes $6 \%$ from $J / \psi$ reconstruction, $2 \%$ for statistical uncertainty in the simulation, $11 \%$ for the $\chi^{2}$ correction, and $3 \%$ for proton reconstruction.

We use world average values [13] for $\mathcal{B}\left(J / \psi \rightarrow e^{+} e^{-}\right)$, $\mathcal{B}\left(J / \psi \rightarrow \mu^{+} \mu^{-}\right)$, and $\mathcal{B}\left(\Lambda \rightarrow p \pi^{-}\right)$.

We estimate the mean expected background in the sig- 

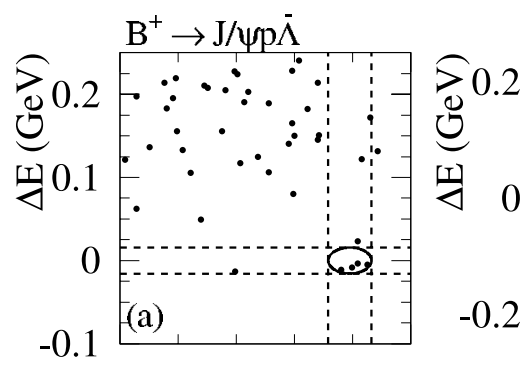

5.2

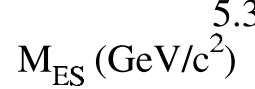

$-0.2$

(d)

5.2 $\mathrm{B}^{0} \rightarrow \mathrm{J} / \psi \mathrm{pp} \overline{\mathrm{p}}$
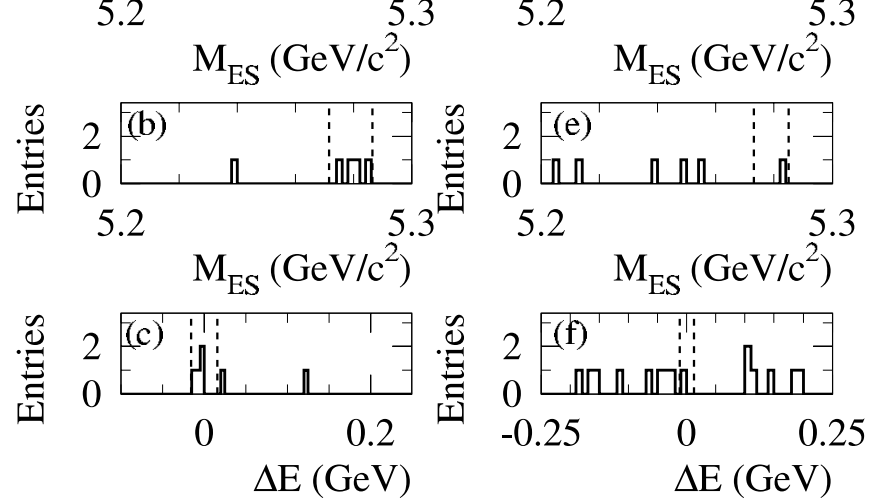

FIG. 2: (a) Distribution of $B^{+} \rightarrow J / \psi p \bar{\Lambda}$ candidates in the $\Delta E-m_{\mathrm{ES}}$ plane, with the signal ellipse and its projection in each dimension (dashed lines). Histogram of candidates within marked bands in (b) $m_{\mathrm{ES}}$ and (c) $\Delta E$. Plots (d)-(f) show similar quantities for $B^{0} \rightarrow J / \psi p \bar{p}$.

nal ellipse $\left(\mu_{B}\right)$ from the number $N_{A}$ elsewhere in the AW: $\mu_{B}=f \cdot N_{A}$. We obtain $f$, the proportionality constant, from a larger sample in which only one proton satisfies the proton identification criteria. We perform a Kolmogorov test [14] to verify that the distribution of candidates in the $\Delta E-m_{\mathrm{ES}}$ plane is similar to the standard selection. Comparing the regions outside the ellipse, the test gives a probability of 0.52 for $B^{+} \rightarrow J / \psi p \bar{\Lambda}$ and 0.36 for $B^{0} \rightarrow J / \psi p \bar{p}$. We obtain $f=0.0054 \pm 0.0035$ $\left(B^{+}\right)$and $f=0.0051 \pm 0.0013\left(B^{0}\right)$. The uncertainties are largely statistical, but include a component $(16 \%$ for $B^{+}$and $2 \%$ for $B^{0}$ ) due to differences in the number of events with multiple candidates.

For $B^{+} \rightarrow J / \psi p \bar{\Lambda}, N_{A}=39$, implying an expected background of $0.21 \pm 0.14$ events. We observe four candidates in the signal ellipse (Fig. 2). The probability of observing $\geq 4$ candidates when expecting $0.21 \pm 0.14$ is $2.5 \times 10^{-4}$. Three of the four are positively charged. Two of the four $J / \psi$ mesons decay to $e^{+} e^{-}$and two to $\mu^{+} \mu^{-}$.

To interpret this result as a $B^{+}$branching fraction $\mathcal{B}$, we undertake a Bayesian analysis with a uniform prior above zero. We define the likelihood for $\mathcal{B}$ as the probability of observing exactly four events, including uncertainties on the expected background, signal efficiency, secondary branching fractions, and number of $\Upsilon(4 S)$ decays, $(88.9 \pm 1.0) \times 10^{6}$. We assume the branching fractions $\mathcal{B}\left(\Upsilon(4 S) \rightarrow B^{+} B^{-}\right)=\mathcal{B}\left(\Upsilon(4 S) \rightarrow B^{0} \bar{B}^{0}\right)=0.5$.

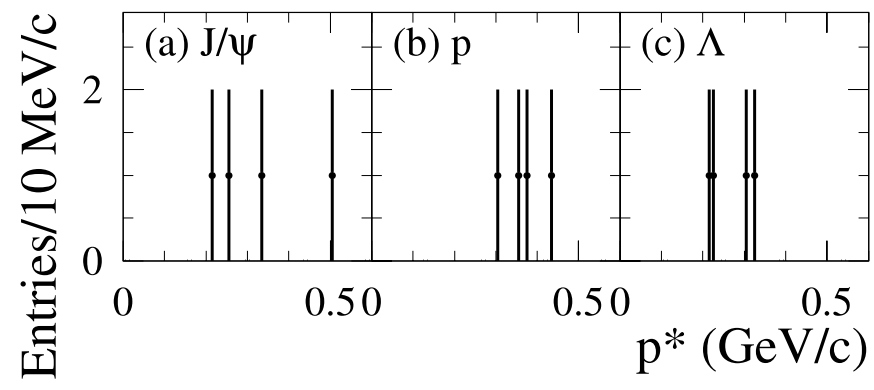

FIG. 3: Momentum in the $B^{+}$rest frame of the (a) $J / \psi$, (b) proton, and (c) $\bar{\Lambda}$ daughters of the four $B^{+} \rightarrow J / \psi p \bar{\Lambda}$ candidates.

The central value for $\mathcal{B}$ is the peak of the likelihood function. We obtain " $\pm 1 \sigma$ " uncertainties from a confidence interval that encloses $68.3 \%$ of the area of the likelihood function, selected such that the likelihoods for all values of $\mathcal{B}$ in the interval are larger than the likelihoods outside. The result is $\mathcal{B}\left(B^{+} \rightarrow J / \psi p \bar{\Lambda}\right)=$ $\left(11.6_{-5.6}^{+8.5}\right) \times 10^{-6}$. We similarly obtain a $90 \%$ CL upper limit of $26 \times 10^{-6}$.

If we consider only the statistical uncertainty, the result would be $\mathcal{B}\left(B^{+} \rightarrow J / \psi p \bar{\Lambda}\right)=\left(11.6_{-5.3}^{+7.4}\right) \times 10^{-6}$. Subtracting these uncertainties in quadrature would indicate contributions from systematic errors of $4.2 \times 10^{-6}$ and $1.8 \times 10^{-6}$ on the upper and lower sides respectively. The systematic error arises almost entirely from the uncertainty on the signal efficiency.

The creation of a narrow QCD exotic bound state as an intermediate resonance in the $B^{+}$decay would be reflected as a narrow $p^{*}$ distribution of the other decay daughter. We do not observe any significant clustering in the $p^{*}$ distributions of the $J / \psi$, proton, or $\bar{\Lambda}$ daughters of the four $B^{+}$candidates (Fig. 3). The resolution in $p^{*}$ is $\sigma \sim 20 \mathrm{MeV} / c$.

For $B^{0} \rightarrow J / \psi p \bar{p}$, there are 126 events outside the signal ellipse, indicating an expected background of $0.64 \pm 0.17$ events, and one event in the ellipse. Following the procedure described for $B^{+} \rightarrow J / \psi p \bar{\Lambda}$, and again assuming a uniform prior above 0 , we obtain $\mathcal{B}\left(B^{0} \rightarrow J / \psi p \bar{p}\right)<1.9 \times 10^{-6}(90 \% \mathrm{CL})$. This limit is dominated by statistical uncertainty.

In summary, we observe four $B^{+} \rightarrow J / \psi p \bar{\Lambda}$ candidates in a data set of $(88.9 \pm 1.0) \times 10^{6} \Upsilon(4 S)$ decays. The probability of the expected charged $B$ background, $0.21 \pm 0.14$ events, producing $\geq 4$ events is $2.5 \times 10^{-4}$. The branching fraction is $\left(12_{-6}^{+\overline{9}}\right) \times 10^{-6}$, where the uncertainty includes both statistical and systematic components. This result can be interpreted as a 90\% CL upper limit of $26 \times 10^{-6}$.

We observe one $B^{0} \rightarrow J / \psi p \bar{p}$ candidate with an expected background of $0.64 \pm 0.17$, and determine a $90 \%$ CL upper limit of $1.9 \times 10^{-6}$ on the branching fraction.

Neither final state makes a significant contribution to 
the observed excess of $J / \psi$ mesons in inclusive $B$ decay. The momentum distributions of the $B^{+}$daughters do not provide evidence for QCD exotic particles produced as narrow intermediate states.

We are grateful for the excellent luminosity and machine conditions provided by our PEP-II colleagues, and for the substantial dedicated effort from the computing organizations that support BABAR. The collaborating institutions wish to thank SLAC for its support and kind hospitality. This work is supported by DOE and NSF (USA), NSERC (Canada), IHEP (China), CEA and CNRS-IN2P3 (France), BMBF and DFG (Germany), INFN (Italy), FOM (The Netherlands), NFR (Norway), MIST (Russia), and PPARC (United Kingdom). Individuals have received support from the A. P. Sloan Foundation, Research Corporation, and Alexander von Humboldt Foundation.

* Also with Università di Perugia, Perugia, Italy

$\dagger$ Also with Università della Basilicata, Potenza, Italy

¥ Also with IFIC, Instituto de Física Corpuscular, CSICUniversidad de Valencia, Valencia, Spain

$\S$ Deceased

[1] CLEO Collab., R. Balest et al., Phys. Rev. D 52, 2661 (1995); S. Chen et al., Phys. Rev. D 63, 031102 (2001).
[2] BABAR Collab., B. Aubert et al., Phys. Rev. D 67, 032002 (2003).

[3] S. Schrenk, in Proceedings of the 30th International Conference on High Energy Physics, edited by C.S. Lim and T. Yamanaka (Osaka, Japan, 2000), Vol. 2, p. 839. See also Fig. 1 of Ref. [6].

[4] M. Beneke, G.A. Schuler, and S. Wolf, Phys. Rev. D 62, 034004 (2000).

[5] T. Sjöstrand, Computer Physics Commun. 82, 74 (1994).

[6] C.-H.V. Chang and W.-S. Hou, Phys. Rev. D 64, 071501 (2001).

[7] G. Eilam, M. Ladisa, and Y.-D. Yang, Phys. Rev. D 65, 037504 (2002).

[8] S.J. Brodsky and F.S. Navarra, Phys. Lett. B 411, 152 (1997).

[9] BABAR Collaboration, B. Aubert et al., Nucl. Instr. and Methods A479, 1 (2002).

[10] G. C. Fox and S. Wolfram, Phys. Rev. Lett. 41, 1581 (1978).

[11] A. Drescher et al., Nucl. Instr. and Methods A237, 464 (1985). See Ref. [2] for implementation.

[12] R. Sinkus and T. Voss, Nucl. Instr. and Methods A391, 360 (1997). See Ref. [2] for implementation.

[13] Particle Data Group, K. Hagiwara et al., Phys. Rev. D 66, 010001 (2002).

[14] A.N. Kolmogorov, Giornale dell'Istituto Ital. degli Attuari 4, 83 (1933); N.V. Smirnov, Bulletin Mathématique de l'Université de Moscou 2, 3 (1939). For implementation, see "Hbook - Statistical Analysis and Histogramming", CERN Program Library entry Y250 (1998). 